\title{
Bilateral anterior tibial compartment syndrome in association with hypothyroidism
}

\author{
A.K. Thacker, Dilip Agrawal and N.B.S. Sarkari \\ Department of Medicine, B.R.D. Medical College, Gorakhpur, 273013 India
}

\begin{abstract}
Summary: Local vascular and traumatic involvement are well-recognized causes of anterior tibial compartment syndrome (ATCS) terminating as myoneural ischaemia. However, in a large number of patients the cause of ATCS remains unidentified. We document the occurrence of bilateral ATCS in a patient of hypothyroidism, to our knowlege a previously unrecorded association.
\end{abstract}

\section{Introduction}

Acute anterior tibial compartment syndrome (ATCS) is a well-defined entity with seemingly diverse aetiologies. ${ }^{1,2}$ The syndrome follows an increase in the compartment pressure, initiating a sequence of self-perpetuating ischaemia, oedema and myoneural damage which, if prolonged, may be irreversible. ATCS in the majority of cases develops after vascular or osseous limb trauma, prolonged limb compression, burns or undue exertion. However, it has also been recorded in association with Buerger's disease, polyarteritis nodosa, hereditary bleeding disorders like haemophilia, eclampsia, ${ }^{3}$ nephrosis, ${ }^{4}$ epilepsy and acute generalized myopathy. We document bilateral ATCS in a florid case of hypothyroidism.

\section{Case report}

A 40 year old male developed sudden severe pain and swelling below the right knee followed by foot drop on the same day. There was no history of heavy exertion, drug or alcohol intake, trauma or snake bite. A release incision was made to decompress the anterior tibial compartment. The preoperative investigations revealed haemoglobin $9.0 \mathrm{~g} / \mathrm{dl}$, white blood count $13.6 \times 10^{9} / 1$ with $13 \%$ eosinophils and ESR $27 \mathrm{~mm} /$ hour. His fasting and post-prandial blood glucose, serum urea, uric acid

Correspondence: A.K. Thacker, M.D., D.M., Assistant Professor of Neurology, B.R.D. Medical College, Gorakhpur, 273013 India.

Accepted: 19 April 1993 and urinalysis were normal. The surgeon noted the presence of excessive boggy, non-pitting, subcutaneous tissue on the release incision.

A fortnight later he developed similar pain and swelling over the left lower limb and it too was followed by foot drop. Closer scrutiny revealed symptoms of progressive weight gain, cold intolerance, apathy and marked snoring in the recent past.

On examination he was a middle-aged overweight man with typical myxoedematous facies. His supine blood pressure was $160 / 90 \mathrm{mmHg}$ and the pulse rate was $70 /$ minute. There was no pallor or thyromegaly. The left lower limb showed marked palpably tense, indurated, brawny swelling below the knee with erythematous discoloration and increased local heat and tenderness. The pulsations of dorsalis pedis were intact. He had bilateral foot drop with MRC grade 0/5 power in the dorsiflexors of the ankles and extensors of the toes. The right lower limb was mildly oedematous and had an infected wound of the release incision. The sensations for fine touch and pinprick over the first interdigital cleft on the dorsum of both the feet were diminished. The ankle jerk on the right side showed delayed relaxation, but could not be elicited on the left side because of swelling. The rest of the systemic examination was normal.

Full blood count, glucose, urea, calcium, phosphorus, iron, electrolytes and urinalysis were within normal limits. The serum triiodothyronine was $0.15 \mathrm{nmol} / 1$ (normal $1.1-2.5 \mathrm{nmol} / \mathrm{l}$ ), thyroxine $10 \mathrm{nmol} / \mathrm{l}(55-135 \mathrm{nmol} / \mathrm{l})$ and serum TSH $118 \mathrm{mU} / 1 \quad(0.5 \mathrm{mU} / \mathrm{l})$. The anti-microsomal antibody and anti-thyroglobulin titres were not 
significantly raised. The electrocardiogram showed low voltage complexes with normal $\mathbf{P} \mathbf{R}$ interval and QRS complexes. The cross-sectional twodimensional echo-cardiography revealed a small posterior pericardial effusion. Electrophysiological studies showed a complete conduction block along the right and left peroneal nerves in the knee-ankle segment. The motor and sensory conductions over the median and ulnar nerves were within normal limits. The electromyography of the right tibialis anterior and extensor digitorum brevis showed increase in polyphasic motor unit potentials with a decreased recruitment phenomena. The electromyography over the left lower limb could not be carried out initially owing to oedema, but subsequently the anterior tibial muscles of the left side also showed features of denervation atrophy.

The patient responded dramatically to thyroid replacement with generally improved well-being. However bilateral foot drop and mild wasting of anterior tibial muscles persisted. The infected wound healed following ofloxacin therapy.

\section{Discussion}

The patient presented with bilateral foot drop secondary to compression of the common peroneal nerves preceded by severe pain in the legs. The associated characteristic swelling of the lower limbs below the knee along with hypoaesthesia and presence of arterial pulsation, even in the absence of a demonstrable rise in the compartment pressure, points to the diagnosis of ATCS. The patient had definite clinical and biochemical parameters of primary hypothyroidism. To our knowledge this association of bilateral ATCS and myxoedema has not been described hitherto.

Bilateral anterior tibial compartment syndrome constitutes approximately $7 \%$ of the reported instances of ATCS. ${ }^{2}$ The ATCS can be initiated by the causes that decrease the size of the compartment or by those increasing its contents. In descending order of frequency these can be grouped as vascular, idiopathic (or exercise induced), traumatic or unclassified. The diverse aetiologies of ATCS defy a universally accepted pathogenetic mechanism. Nevertheless, the presence of arterial diseases and/or trauma in a majority of these patients with the frequent demonstration of ischaemic muscular necrosis ${ }^{5}$ point to a decrease in the arterial perfusion as the primary event. Slud- 3

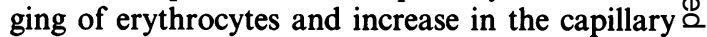
permeability elevate the pressure within a rigid $\subseteq$ cylindrical osseofascial space. A synergistic vicious $\vec{F}$ cycle of myoneural ischaemia is generated which, if $\frac{+}{-}$ prolonged, terminates as muscle necrosis. ${ }^{5-7}$

In myxoedema several factors may be conducive $\frac{\bar{\sigma}}{\bar{c}}$ to the development of ATCS. Thus an increase in $\overrightarrow{\mathbb{D}}$ the compartment contents may result from inter- $\varrho$ sitial oedema and in some cases from true muscle के hypertrophy. Increase in the capillary permeability $\overrightarrow{0}$ and slow lymphatic drainage can lead to extravasa- $\overrightarrow{-}$ tion of protein-rich fluid in the interstitium, $\vec{\omega}$ somewhat akin to serous effusions of myxoedema. ${ }^{8} \frac{}{\circ}$ Naeye ${ }^{9}$ reported deposition of an amorphous $\frac{0}{3}$ material staining as glycosaminoglycan in the wallsof alveolar capillaries, pulmonary veins and $\subseteq$ glomeruli of hypothyroid patients. A similar $\stackrel{\infty}{=}$ deposition in the compartment vessels may lead to ${ }^{\circ}$ decreased nutritive perfusion, which has been dem- $\stackrel{\infty}{\rightarrow}$ onstrated in the idiopathic form of ATCS on 0 radioisotope flow studies. ${ }^{10}$

Skeletal muscle hypertrophy occurs in $1 \%$ of cases of myxoedema myopathy ${ }^{11}$ and is referred to as Hoffman's syndrome in adults ${ }^{12}$ and as Kocher $\frac{\Phi}{3}$ Debre-Semelaigne syndrome in infants and children. Electron microscopy also reveals excess glycogen in the membrane-bound space with dis $\vec{\odot}$ ruption of the sarcolemma and hypertrophis muscle fibres. ${ }^{13}$

Similarly decrease in the compartment size owing to connective tissue proliferation in myxoedema may be another contributory factor. It is postulated that stimulation of fibroblasts by thyroid stimulating hormone and diminished deg- $\stackrel{2}{\Rightarrow}$ radation of tissue hyaluronate owing to thyroxine lack alter the collagen-glycosaminoglycan ratio leading to increase in the connective tissues contents. ${ }^{14}$ Experimental studies have shown that $40 \%$ less volume is required to elevate intracompartmental pressures to equivalent levels in casted compared with uncasted hindlimb antero-lateral compartment in dogs. ${ }^{15}$

Hence it may be assumed that the vascular, muscular and connective tissue abnormalities can $\circ$ predispose a patient with myxoedema to ATCS. However, as in most instances with idiopathic ATCS, what initiates the process in hypothyroidism is difficult to surmise.

\section{References}

1. Bradley, E.L. The anterior tibial compartment syndrome. Surg Gynecol Obstet 1973, 136: 289-297.

2. Mubarak, S.J. \& Hargens, A.R. Acute compartment syndromes. Surg Clin North Am 1983, 63: 539-565.

3. Manson, I.W. Post partum eclampsia complicated by the anterior tibial syndrome. $\mathrm{Br}$ Med J 1964, 2: 1117.

4. Sweeney, H.E. \& O'Brien, F. Bilateral anterior tibial syndrome in association with the nephrotic syndrome; report of a case. Arch Intern Med 1965, 116: 487.

5. Harman, J.W. The significance of local vascular phenomena in the production of ischemic necrosis in skeletal muscle. Am J Pathol 1948, 24: 625. 
6. Mortez, W.H. The anterior compartment ischemia syndrome. Am Surg 1953, 19: 729.

7. Whitesides, T.E., Jr, Hirada, H. \& Marimoto. K. Compartment syndromes and the role of fasciotomy. Its parameters and techniques. Am Acad Orthop Surg Instructional Course Lectures 1977, 26: 179-194.

8. Parving, H., Hansen, J.M., Nilsen, S.V., Rossing, N., Munck, O. \& Lassen, N.A. Mechanisms of edema formation in myxedema - increased protein extra-vasation and relatively slow lymphatic drainage. $N$ Engl J Med 1981, 301: 460.

9. Naeye, R.L. Capillary and venous lesions in myxedema. Lab Invest 1963, 12: 465-470.

10. Freedman, B.J. \& Knowles, C.H.R. Anterior tibial syndrome due to arterial embolism and thrombosis. Br Med J 1959, 2: 270.
11. Ramsay, I.D. Endocrine myopathies. Practitioner 1982, 226: 1075-1080.

12. Klein, I., Parker, M., Shebert, R. et al. Hypothyroidism presenting as muscle stiffness and pseudo-hypertrophy: Hoffman's syndrome. Am J Med 1981, 70: 891-894.

13. Pencea, V., Cotrutz, C., Dobrescu, G., Galasuna, C., Pencea, I.I. \& Dudeanu, I. Histopathological data on six cases of hypothyroidism accompanying muscular chronic disease. Rev Roumanie Med Endrocrinol 1985, 23: 105-109.

14. Bland, J.H. \& Frymoyer, J.W. Rheumatic syndromes of myxedema. $N$ Engl J Med 1970, 282: 1171-1174.

15. Garfin, S.R., Mubarak, S.J. \& Evans, K.L. Quantification of intra compartmental pressure and volume under plaster casts. J Bone Joint Surg 1981, 63A: 449-453.

\title{
Carcinoma of the breast at the site of migrated pacemaker generators
}

\author{
D.S. Bhandarkar, A.D.M. Bewu' and T.V. Taylor \\ Departments of Surgery and ${ }^{1}$ Cardiology, Manchester Royal Infirmary, Oxford Road, Manchester \\ $M 139 W L, U K$
}

\begin{abstract}
Summary: We report two patients in whom breast malignancy developed in the proximity of 'migrated' pacemaker generators. These cases and the similar ones reported in the literature raise concerns whether this association is merely coincidental or whether the pacemaker generator is responsible for the occurrence in some inexplicable manner. We urge for a routine, careful examination in all patients with implanted pacemaker generators at follow-up visits. This would help in (a) timely detection of migration of the pacemaker generators and (b) earlier diagnosis of any mass developing close to the migrated generators.
\end{abstract}

\section{Introduction}

We report, in two women, the development of breast cancer in the proximity of pacemaker generators, a rare association. Both the generators had migrated to a sub-mammary position from their original sites of implantation.

\section{Case reports}

Case 1

During a follow-up visit to the pacemaker clinic an 83 year old woman was detected to have a lump in

Correspondence: T.V. Taylor, M.D., Ch.M., F.R.C.S. (Eng.), F.R.C.S.(Ed.)

Accepted: 16 March 1993 her left breast. Thirteen years previously she underwent implantation of a Cordis Omni pacemaker to treat symptomatic sinus bradycardia and cardiac pauses. The pacemaker generator was changed to a Cordis 337A VVI type 6 years later. On both these occasions the generators were placed in left infraclavicular pockets. The lump noticed was situated in the outer, lower quadrant, was firm, ill-defined and free from the underlying structures. The overlying skin was tethered to it. The axillary or supraclavicular nodes were not palpable and the abdominal examination was unremarkable. Serum biochemical parameters were within normal limits. A plain X-ray of the chest (Figure 1) showed the pacemaker generator to be lying over the ribcage on the left side. This site corresponded with the clinically palpable lump. A fine-needle aspirate diagnosed it to be an adenocarcinoma. In view of 